\title{
Cancer as an immune-mediated disease
}

\author{
This article was published in the following Dove Press journal: \\ ImmunoTargets and Therapy \\ 13 June 2012 \\ Number of times this article has been viewed
}

\section{Michael R Shurin \\ Departments of Pathology and Immunology, University of Pittsburgh Medical Center, Pittsburgh, PA, USA}

Correspondence: Michael R Shurin UPMC, Pathology, Scaife Hall S735, 3550

Terrace Street, Pittsburgh, PA I526I, USA

Tel + I 4I2648 984 I

Fax + | 4I2-648 8I58

Email shurinmr@upmc.edu
Abstract: The link between oncology and immunology has a long history and its development is forced by the necessity to develop innovative and highly efficient modalities for immunological destruction of malignant cells. The limited efficacy of surgery, chemotherapy and radiation also exemplify these issues, as these treatments do not eliminate all cancerous cells, do not address the immunosuppressive nature of the disease and can further impair the patient's immune response weakening patient's resistance to the cancer. Multidisciplinary analysis of the interaction between the immune system and cancer in preclinical and clinical settings suggests that the immune system is closely intertwined with both cancer pathogenesis and treatment. On the one hand, cancer is a manifestation of malfunctions in immunity, as malignant cells manage to escape recognition and elimination by the immune system. Chronic infections and inflammation associated with limited or polarized immune responses also contribute to carcinogenesis and tumor progression. The tumor immunoenvironment represents specific conditions and elements that support cancerous cell survival, proliferation and spreading. On the other hand, the specificity and strength of antitumor immunity is a powerful and efficient tool that can be used to recognize and destroy neoplastic cells or their supporting microenvironment. Understanding the role of the immune system in controlling and supporting tumor initiation, formation, growth and progression has crucial implications for cancer therapy and will therefore guide the future development of cancer immunotherapy and its combination with conventional therapies to achieve optimal antitumor effects in patients with different types of cancer.

Keywords: tumor immunology and immunotherapy, tumor immunoenvironment, cancer, immunosuppression, regulatory immune cells

The field of immunology encompasses nearly all disciplines in modern medicine, including infectious, pulmonary, cardiovascular, connective tissue, neurological, endocrine, reproductive, psychological, transplantation, cancer, and many other diseases. Many human diseases stem from immunologic or inflammatory factors, as more than $25 \%$ of patients admitted to hospitals suffer from some form of immune-mediated inflammatory condition. ${ }^{1}$ Cancer represents more than 200 different diseases and the World Health Organization considers it to be one of the most important medical conditions facing mankind in the 21 st century. More than 11 million people are diagnosed with cancer every year, with 8 million deaths worldwide. Cancer is likely to become the most prevalent cause of death as diagnostics, health care, lifestyle, diet, and life expectancy continue to improve. Although the ability of the immune system to effectively respond to tumor growth is now recognized, the role of the immune system in controlling tumor initiation and expansion as well as cancer progression has been controversial for some time. submit your manuscript $\mid$ www.dovepress.com

Dovepress

http://dx.doi.org// 0.2147/ITT.S29834
ImmunoTargets and Therapy 2012:I I-6

(C) 2012 Shurin, publisher and licensee Dove Medical Press Ltd. This is an Open Access article which permits unrestricted noncommercial use, provided the original work is properly cited. 
Understanding how the immune system affects cancer development and progression is one of the most challenging questions in immunology. The surgeon to the Duke of Kent injected himself with malignant tissue as a prophylaxis against developing cancer in 1777 , and the doctor to Louis 17 inoculated himself with breast cancer in hopes of reversing soft-tissue sarcoma in 1808. However, the principle that the immune system can recognize and respond to neoplastic cells was first proposed in the 19th century. In 1891, William Coley, a surgeon from the Memorial Sloan Kettering Cancer Institute in New York, reported that using heat-killed endotoxin-containing bacteria (a combination of Streptococcus pyogenes and Serratia marcescens) resulted in a cure rate of $10 \%$ in soft-tissue sarcoma patients. ${ }^{2}$ In 1909, Paul Ehrlich suggested that the immune system surveys the body and prevents the outgrowth of carcinomas that would otherwise occur with high frequency. ${ }^{3}$ With an increased understanding of immune response mechanisms, Frank Macfarlane Burnet proposed that tumor-specific neoantigens were capable of eliciting protective immunity. ${ }^{4}$ Lewis Thomas speculated that complex organisms with long lifespans should possess mechanisms capable of protecting against tumors. ${ }^{5}$

These initial hypotheses and postulates were confirmed through numerous experimental model systems used to demonstrate that the immune system can identify and destroy cancerous cells in a process termed cancer immunosurveillance, an important defense pathway against cancer. Furthermore, reports of increased incidence and aggressiveness of a variety of cancers in patients receiving immunosuppressive therapy or in immunodeficient patients support the hypothesis that the immune system plays a critical role in controlling malignant tumor generation. For instance, a systematic review of observational studies evaluating cancer incidence in both organ recipients and people with human immunodeficiency virus/acquired immunodeficiency syndrome (HIV/AIDS) suggested that a weakened immune system increases the cancer risk in immunocompromised populations. ${ }^{6}$ The ability of immune cells to recognize and destroy cancerous cells both in vitro and in vivo suggests the presence of cellular mechanisms for tumor immunosurveillance. Thus, data obtained from various studies involving rodent models of cancer and patients with cancer offer compelling evidence that particular innate and adaptive immune cell types, specific effector molecules, and definite pathways can collectively function as extrinsic tumor-suppressor mechanisms. ${ }^{7}$

Understanding the role of the immune system in recognizing and eliminating tumors and identifying several classes of tumor-associated antigens stimulated the discipline of tumor immunology, with special emphasis on novel immunotherapeutic approaches to treating cancer. Based on successful prevention of cancer development and elimination or inhibition of tumor growth in preclinical animal models, various therapeutic vaccines have been designed and tested in clinical trials. ${ }^{8}$ These strategies have included the use of tumor cell lysates and proteins, dendritic cells (DCs) and T cells, cytokines, Toll-like receptor ligands, immunomodulators, antibodies, specific DNA, and other molecules administered to several different tissues, including intratumoral or systemic injections and injection into lymph nodes, skin, subcutaneous tissue, and muscle. Unfortunately, the results of these clinical trials were disappointing. Although immunotherapy of cancer is still considered an attractive therapeutic approach, its impact on clinical practice, with the exception of several antibodies, cytokines, and dendritic cell vaccines, is limited. ${ }^{9}$ Most clinically apparent cancers grow relentlessly, despite measurable immune responses, suggesting that the antitumor immunity is, or becomes, ineffective. This also suggests that though the immune system controls nascent cancer through immunosurveillance, tumors can escape this control and develop into clinical cancer.

Cancers have been found to utilize diverse mechanisms to avoid, suppress, and polarize both innate and adaptive antitumor immune responses. Immune escape results from tumorinduced changes in cancerous cells as well as in surrounding stromal tissues and immune system. Downregulation of antigen processing and presentation by malignant cells, altered expression of certain chemokines and cytokines, induction of apoptosis in immune cells, and suppression of immune cell function have been implicated in tumor escape from immune recognition and elimination. ${ }^{7,-11}$ Importantly, both adaptive and innate responses may be dysfunctional in the tumor microenvironment. For instance, several tumorderived factors have reportedly block generation of DC and their ability to uptake, process, and present tumor antigens to $\mathrm{T}$ cells. ${ }^{10}$ Furthermore, upregulation of the immunosuppressive cell surface glycoprotein CD200 on acute myeloid leukemia cells specifically compromises natural killer (NK) cell antitumor responses. Patients with high CD200 expression on their acute myeloid leukemia cells exhibited a reduced number of activated NK cells and lowered lytic activity and interferon y response against autologous CD200-expressing leukemic cells. ${ }^{12,13}$

Additional studies have revealed that polarization of immune response in the tumor environment and tumormediated accumulation of immunosuppressive regulatory cells provides another crucial mechanism for tumor escape 


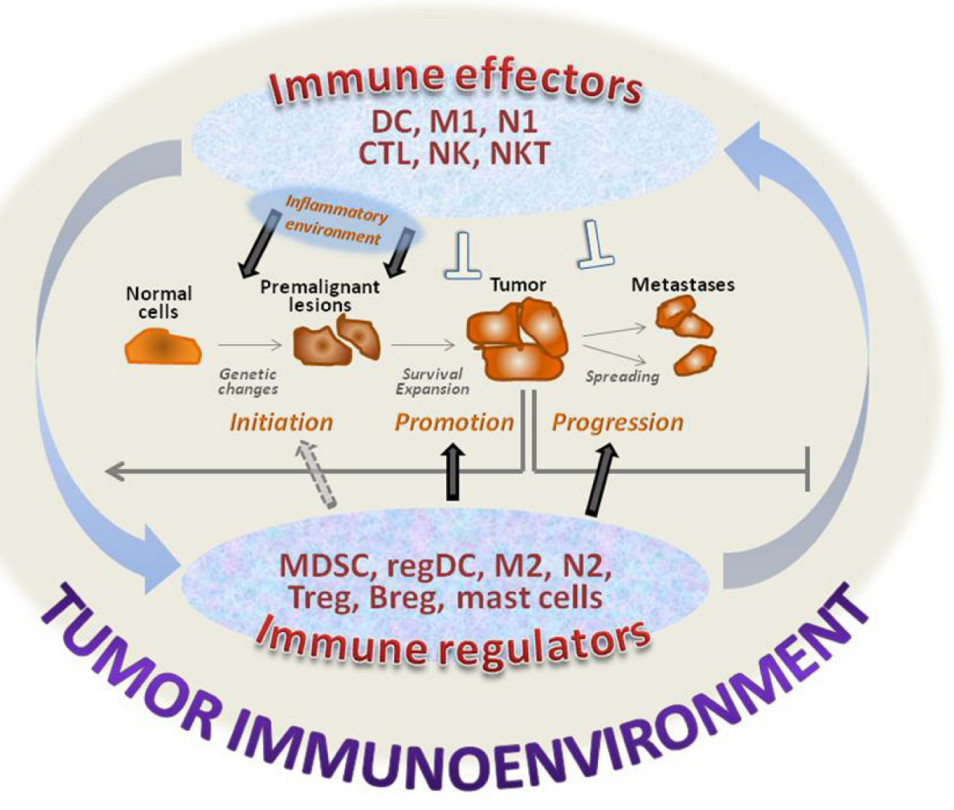

Figure I Cancer is an immune-mediated disease.

Notes: Immune system cells participate in all stages of tumorigenesis and immunosurveillance. For instance, the inflammatory microenvironment contributes to tumor initiation through mutations, genomic instability, and epigenetic modifications. ${ }^{32}$ DC can mediate induction of genomic instability. ${ }^{36}$ Inflammatory cytokines may also induce proliferation of premalignant cells and form a microenvironment in which premalignant cells can survive, expand, and accumulate additional mutations and epigenetic changes. Innate and adaptive immune effector cells control cancerous cell growth and progression by inducing and maintaining anti-tumor immune responses and by suppressing intratumoral angiogenesis. ${ }^{37}$ However, tumor-derived factors direct the differentiation of immune cells and attract different subsets of immune regulatory cells in the tumor microenvironment. Immune regulators, including MDSC, regDC, regulatory T and B cells, alternatively activated M2 and N2, as well as mast cells, display profound protumorigenic properties and support tumor cell proliferation, spreading, survival of metastases, and stimulate neoangiogenesis. ${ }^{41}$ Additionally, tumor-derived factors prevent re-polarization of differentiation of immune regulators into immune effector cells. Thus, the tumor immunoenvironment includes unique and dynamically evolving subpopulations of immune cells and factors that provide supporting and inhibitory stimuli for tumor formation and progression. The balance between these bi-directorial immune forces is tightly regulated by multiple intrinsic and extrinsic factors and conditions, such as tumor- and stroma-derived cytokines, chemokines and growth factors, chronic inflammatory diseases of the host, medications and treatments, mental and physical stress, age-associated conditions, and other factors.

Abbreviations: DC, dendritic cells; MDSC, myeloid-derived suppressor cells; regDC, regulatory DC; M2, macrophage type 2; N2, neutrophil type 2.

and may explain the low efficacy of many immunotherapeutic approaches. Several subsets of immune regulatory cells have been identified and characterized. Their roles in inhibiting antitumor immunity and maintenance of tumor tolerance and unresponsiveness have been demonstrated both in animal models and in humans with cancer. These cells include several subpopulations of regulatory $\mathrm{T}$ cells (Treg), myeloid-derived suppressor cells (MDSCs), regulatory dendritic cells, alternatively activated tumor-associated macrophages, neutrophils, mast cells, regulatory B cells, and possibly specific subsets of natural killer T cells. ${ }^{14-18}$

Tumor-redirected differentiation and functional polarization of immune cells results in accumulation of specific immune cell subsets with protumorigenic potential, which support tumor development, growth, and progression through various mechanisms. Thus, we now understand that the immune system plays a dual role in cancer progression: it can not only suppress tumor growth by destroying cancer cells or inhibiting their outgrowth, but also promote tumor progression either by selecting for tumor cells that are more fit to survive in an immunocompetent host or by establishing conditions within the tumor microenvironment that facilitate tumor outgrowth. ${ }^{11}$ For instance, antigen-specific Treg cells primarily target DCs and inhibit DC functions, including expression of costimulatory molecules and presentation of antigens early during immune response generation. The end result is inhibition of both expansion and differentiation of T effector cells. Polyclonal Treg cells also act on DCs, but at a later phase, and do not inhibit expansion of T effector cells but appear to modulate differentiation and cell trafficking. ${ }^{19}$ MDSCs also exert their direct immunosuppressive effects on antigen-specific $\mathrm{T}$ cell responses, but can also act upon mitogen-activated $\mathrm{T}$ lymphocytes, therefore bypassing antigen dependency. ${ }^{20}$ In addition to being potently suppressing $\mathrm{T}$ cell function, recent studies have demonstrated the ability of MDSCs to modulate the activities of NK and myeloid cells and have implicated MDSCs in induction of regulatory $\mathrm{T}$ cells. ${ }^{21}$ Regulatory DCs in cancer may directly and indirectly maintain antigen-specific and nonspecific $\mathrm{T}$ cell unresponsiveness by controlling $\mathrm{T}$ cell polarization, MDSC and Treg cell differentiation, and their activities and by affecting specific microenvironmental conditions in premalignant 
niches. ${ }^{22}$ Tumor-associated macrophages (TAMs) are also significant in fostering tumor progression. The protumor properties of TAMs derive from regulation of angiogenic programming, production of soluble mediators that support proliferation, survival and invasion of malignant cells, and direct and indirect suppression of cytotoxic T cell activity. ${ }^{17,23}$ TAMs also contribute to immune evasion by inducing tolerogenic forkhead box $\mathrm{P} 3$ and interleukin 10-secreting T cells as well as by upregulating inhibitory receptor cytotoxic T lymphocyte antigen-4 expression in effector T cells. ${ }^{24}$

Although neutrophils are traditionally described in the context of their antibacterial functions, increasing evidence has demonstrated that tumor-associated neutrophils are also important in cancer biology. ${ }^{25}$ Many cancers can recruit neutrophils to sites of tumorigenesis where they enhance tumor growth. ${ }^{26}$ Type 2 neutrophils can inhibit effector $\mathrm{T}$ cell functions; a suggested mechanism for the suppression of $\mathrm{T}$ cell proliferation and responsiveness to stimulation is through secretion of stored arginase 1, which degrades extracellular arginine, a factor needed for the proper activity of T cells. ${ }^{25}$ Additionally, products secreted from tumor-associated neutrophils, such as reactive oxygen species and proteinases, have defined and specific roles in regulating tumor cell proliferation, angiogenesis, and metastasis. ${ }^{18}$

Neutrophils can also significantly impact the tumor microenvironment by producing cytokines and chemokines, which influence inflammatory cell recruitment and activation; additionally, the role of inflammation in carcinogenesis has become increasingly evident. ${ }^{27} \mathrm{~A}$ pathophysiological association between inflammation and cancer was proposed in the nineteenth century, when in 1863 Rudolf Ludwig Karl Virchow observed leucocytes in neoplastic tissues and attempted to correlate inflammation and cancer. ${ }^{28}$ He suggested that the "lymphoreticular infiltrate" reflected the origin of cancer at sites of chronic inflammation. Later, numerous laboratory and population-based studies suggested that certain malignancies arise at tissues severely damaged by chronic inflammation. For example, cancers of the stomach, liver, gallbladder, prostate, and pancreas are causally linked to gastric inflammation, chronic hepatitis, cholecystitis, inflammatory atrophy of the prostate, and chronic pancreatitis, respectively. ${ }^{29}$ Colitis, a condition characterized by persistent colonic mucosal inflammation, often progresses to colorectal cancer; inflammatory bowel disease increases the risk of colorectal cancer by 10 -fold, while the management of colitis with anti-inflammatory therapy reduces this risk. ${ }^{30}$ Although approximately $25 \%$ of all cancers have a demonstrated etiologic background of chronic inflammation and/or infection, ${ }^{31} 90 \%-95 \%$ of neoplasias are linked to obesity, tobacco smoke, environmental pollutants, radiation, and chronic infections, which commonly involve a chronic inflammatory state. ${ }^{32}$

The role of inflammation in tumorigenesis is well-known; an inflammatory microenvironment is likely an important cofactor in the development of all tumors, including those in which a direct causal relationship with inflammation has not yet been confirmed..$^{33}$ Inflammatory responses play decisive roles at different stages of cancer development, including initiation, survival, promotion, spreading, and metastasis. Macrophages, neutrophils, mast cells, and DCs are involved in the protumorigenic properties of chronic inflammation and tumor promotion through various mechanisms and pathways. For instance, neutrophils have a dual role in tumor initiation and may alter the extracellular matrix and local microenvironment. Among key mediators involved in tumorigenesis is matrix metalloprotease-9, which regulates oncogene-induced keratinocyte hyperproliferation and progression towards invasive cancer. ${ }^{34}$ Additionally, neutrophil-derived matrix metalloprotease-9 may prevent tumor cell apoptosis. ${ }^{35} \mathrm{KC} / \mathrm{CXCL} 1$, CXCL8, and CCL-3, secreted from neutrophils, may support tumor growth by recruiting leukocytes. Neutrophils may also affect tumor growth by secreting neutrophil elastase, which enters tumor cells and binds insulin receptor substrate-1 to allow increased Akt activation. ${ }^{25}$

In summary, the immune system, in addition to tumorsuppressive functions that eliminate nascent transformed tumor cells, can facilitate tumor initiation and progression by providing a proper tumor microenvironment by maintaining a chronic inflammatory state in the tumor mass and by providing polarized immunosuppressive regulatory cells. Together, the dual host-protective and tumor-promoting actions of immunity are referred to as cancer 'immunoediting' or 'immunosculpting' (ie, cross-talk between immune and tumor cells changing the phenotype of tumor immunobiology). ${ }^{11}$ However, the distinctions between tumor-promoting inflammation and tumor-suppressive immunity are not yet clear due to the dual roles of some cytokines and other immune system molecules. ${ }^{33}$ Furthermore, although the tumor immunoenvironment is now implicated in regulating tumor growth, whether the immune system can directly alter tumor genetics is unknown, despite speculations that reactive oxygen species and reactive nitrogen intermediates produced by inflammatory cells may cause mutations in neighboring epithelial cells to elevate intracellular reactive oxygen species and reactive nitrogen intermediates in 
premalignant cells. ${ }^{32}$ Recently, it was shown that interaction between tumor cells and DCs, but not monocytes, rapidly induces genomic mutator activation-induced cytidine deaminase (AID) and AID-dependent DNA double-strand breaks in tumor cell lines and in primary tumor cells. ${ }^{36}$ AID-mediated genomic damage leads to altered tumorigenicity and indolent behavior of tumor cells in vivo. These data show a novel pathway for immune cells to regulate genomic integrity. ${ }^{36}$

Understanding the role of the immune system in controlling and supporting tumor initiation, formation, growth, and progression has crucial implications for cancer therapy because direct or indirect immunomodulatory interventions aimed at early pathogenic events may no longer be efficient when these pathways have altered due to a varied immune response. Therefore, it is critical to recognize why and how cancer-associated immune activities evolve over time so that time-dependent therapies may be rationally implemented to improve clinical outcome. These new insights regarding the evolving interactions of different cell subsets in the tumor immunoenvironment are constantly improving the design and efficacy of modern cancer immunotherapy protocols, as described elsewhere. ${ }^{37-40}$ Understanding interactions between immune cells, malignant cells, stromal elements, and treatment modalities will therefore guide the future combination of immunotherapy with conventional therapies to achieve optimal anti-tumor effects in patients with different types of cancers.

\section{Acknowledgments}

This work was supported in part by National Institutes of Health RO1 CA154369.

\section{Disclosure}

The author reports no conflicts of interest in this work.

\section{References}

1. Shurin MR, Smolkin YS. Immune-mediated diseases: where do we stand? Adv Exp Med Biol. 2007;601:3-12.

2. Coley WB. II. Contribution to the Knowledge of Sarcoma. Ann Surg. 1891;14(3):199-220.

3. Ehrlich P. Über den jetzigen Stand der Karzinomforschung. Beiträge zur experimentellen Pathologie und Chemotherapie. 1909:117-164.

4. Burnet M. Cancer; a biological approach. I. The processes of control. Br Med J. 1957;1(5022):779-786.

5. Thomas L. Discussion. In: Lawrence HS, ed. Cellular and Humoral Aspects of the Hypersensitive States. New York, NY: Hoeber-Harper; 1959:529-532.

6. Cobucci RN, Saconato H, Lima PH, et al. Comparative incidence of cancer in HIV-AIDS patients and transplant recipients. Cancer Epidemiol. 2012;36(2):e69-e73.

7. Vesely MD, Kershaw MH, Schreiber RD, Smyth MJ. Natural innate and adaptive immunity to cancer. Annu Rev Immunol. 2011;29:235-271.
8. Mellman I, Coukos G, Dranoff G. Cancer immunotherapy comes of age. Nature. 2011;480(7378):480-489.

9. Prestwich RJ, Errington F, Hatfield P, et al. The immune system - is it relevant to cancer development, progression and treatment? Clin Oncol (R Coll Radiol). 2008;20(2):101-112.

10. Shurin MR, Shurin GV, Lokshin A, et al. Intratumoral cytokines/ chemokines/growth factors and tumor infiltrating dendritic cells: friends or enemies? Cancer Metastasis Rev. 2006;25(3):333-356.

11. Schreiber RD, Old LJ, Smyth MJ. Cancer immunoediting: integrating immunity's roles in cancer suppression and promotion. Science. 2011; 331(6024):1565-1570.

12. Coles SJ, Wang EC, Man S, et al. CD200 expression suppresses natural killer cell function and directly inhibits patient anti-tumor response in acute myeloid leukemia. Leukemia. 2011;25(5):792-799.

13. Lion E, Willemen Y, Berneman ZN, Van Tendeloo VF, Smits EL. Natural killer cell immune escape in acute myeloid leukemia. Leukemia. March 26, 2012. [Epub ahead of print.]

14. Byrne WL, Mills KH, Lederer JA, O'Sullivan GC. Targeting regulatory $\mathrm{T}$ cells in cancer. Cancer Res. 2011;71(22):6915-6920.

15. Montero AJ, Diaz-Montero CM, Kyriakopoulos CE, Bronte V, Mandruzzato S. Myeloid-derived suppressor cells in cancer patients: a clinical perspective. J Immunother. 2012;35(2):107-115.

16. Shurin GV, Ouellette CE, Shurin MR. Regulatory dendritic cells in the tumor immunoenvironment. Cancer Immunol Immunother. 2011;61(2):223-230.

17. Allavena P, Mantovani A. Immunology in the clinic review series; focus on cancer: tumour-associated macrophages: undisputed stars of the inflammatory tumour microenvironment. Clin Exp Immunol. 2012;167(2): $195-205$.

18. Gregory AD, Houghton AM. Tumor-associated neutrophils: new targets for cancer therapy. Cancer Res. 2011;71(7):2411-2416.

19. Shevach EM. Biological functions of regulatory T cells. Adv Immunol. 2011;112:137-176.

20. Solito S, Bronte V, Mandruzzato S. Antigen specificity of immune suppression by myeloid-derived suppressor cells. J Leukoc Biol. 2011; 90(1):31-36.

21. Condamine T, Gabrilovich DI. Molecular mechanisms regulating myeloid-derived suppressor cell differentiation and function. Trends Immunol. 2011;32(1):19-25.

22. Ma Y, Shurin GV, Gutkin DW, Shurin MR. Tumor associated regulatory dendritic cells. Semin Cancer Biol. March 6, 2012. [Epub ahead of print.]

23. Chen J, Yao Y, Gong C, Yu F, Su S, Chen J. CCL18 from tumor-associated macrophages promotes breast cancer metastasis via PITPNM3. Cancer Cell. 19(14):541-555.

24. Daurkin I, Eruslanov E, Stoffs T, et al. Tumor-associated macrophages mediate immunosuppression in the renal cancer microenvironment by activating the 15-lipoxygenase-2 pathway. Cancer Res. 2011;71(20):6400-6409.

25. Fridlender ZG, Albelda SM. Tumor-associated neutrophils: friend or foe? Carcinogenesis. 2012;33(5):949-955.

26. Houghton AM. The paradox of tumor-associated neutrophils: fueling tumor growth with cytotoxic substances. Cell Cycle. 2010;9(9): 1732-1737.

27. Kundu JK, Surh Y-J. Emerging avenues linking inflammation and cancer. Free Rad Biol Med. 2012;52(9):2013-2037.

28. Virchow R. Die Krankhaften Geschwülste. First Edition. Berlin: August Hirschwald; 1863.

29. Aggarwal BB, Vijayalekshmi RV, Sung B. Targeting inflammatory pathways for prevention and therapy of cancer: short-term friend, long-term foe. Clin Cancer Res. 2009;15(2):425-430.

30. Kundu JK, Surh YJ. Emerging avenues linking inflammation and cancer. Free Radic Biol Med. 2012;52(9):2013-2037.

31. Hussain SP, Harris CC. Inflammation and cancer: an ancient link with novel potentials. Int J Cancer. 2007;121(11):2373-2380. 
32. Grivennikov SI, Greten FR, Karin M. Immunity, inflammation, and cancer. Cell. 2010;140(6):883-899.

33. Chow MT, Moller A, Smyth MJ. Inflammation and immune surveillance in cancer. Semin Cancer Biol. 2012;22(1):23-32.

34. Coussens LM, Werb Z. Inflammation and cancer. Nature. 2002; 420(6917):860-867.

35. Acuff HB, Carter KJ, Fingleton B, Gorden DL, Matrisian LM. Matrix metalloproteinase-9 from bone marrow-derived cells contributes to survival but not growth of tumor cells in the lung microenvironment. Cancer Res. 2006;66(1):259-266.

36. Koduru S, Wong E, Strowig T, et al. Dendritic cell-mediated activationinduced cytidine deaminase (AID)-dependent induction of genomic instability in human myeloma. Blood. 2012;119(10):2302-2309.
37. Vanneman M, Dranoff G. Combining immunotherapy and targeted therapies in cancer treatment. Nat Rev Cancer. 2012;12(4):237-251.

38. Weiner LM, Murray JC, Shuptrine CW. Antibody-based immunotherapy of cancer. Cell. 2012;148(6):1081-1084.

39. Rosenberg SA. Raising the bar: the curative potential of human cancer immunotherapy. Sci Transl Med. 2012;4(127):127ps128.

40. Pardoll D, Drake C. Immunotherapy earns its spot in the ranks of cancer therapy. J Exp Med. 2012;209(2):201-209.

41. Gabrilovich DI, Ostrand-Rosenberg S, Bronte V. Coordinated regulation of myeloid cells by tumours. Nat Rev Immunol. 2012;12(4):253-268.

\section{Publish your work in this journal}

ImmunoTargets and Therapy is an international, peer-reviewed open access journal focusing on the immunological basis of diseases, potential targets for immune based therapy and treatment protocols employed to improve patient management. Basic immunology and physiology of the immune system in health, and disease will be also covered. In addition, the journal will focus on the impact of manage-

\section{Dovepress}

ment programs and new therapeutic agents and protocols on patient perspective such as quality of life, adherence and satisfaction. The manuscript management system is completely online and includes a very quick and fair peer-review system, which is all easy to use. Visit http://www.dovepress.com/testimonials.php to read real quotes from published authors.

Submit your manuscript here: http://www.dovepress.com/immunotargets-and-therapy-journal 